\title{
Collaborative Rural Healthcare Network: A Conceptual Model
}

\author{
$\underline{\text { doi:10.3991/ijim.v5i3.1669 }}$ \\ U. Raja, D. J. McManus, J.M. Hardin and B. C. Haynes \\ The University of Alabama, Tuscaloosa, USA
}

\begin{abstract}
Healthcare is a critical issue in rural communities throughout the world. Provision of timely and cost effective health care in these communities is a challenge since it is coupled with a lack of adequate infrastructure and manpower support. Twenty percent of the United States of America's population resides in rural communities, i.e., 59 million people; however, only nine percent of the nation's physicians practice in rural communities. Shortage of health care personnel and the lack of equipment and facilities often force rural residents to travel long distances to receive needed medical treatment. Researchers and practitioners are in search of solutions to address these unique challenges. In this research, we present a proposed collaborative model of a health information system for rural communities and the challenges and opportunities of this global issue.
\end{abstract}

Index Terms-Information Systems, Information Technology, Rural Health, Telemedicine.

\section{INTRODUCTION}

Twenty percent of the United States of America's population resides in rural communities, i.e., 59 million people [1]; however, only nine percent of the nation's physicians practice in rural communities [2, 3]. Most of the healthcare information systems applications are designed and implemented in urban settings. Although the core functionality of these systems when implemented in rural communities remains the same, the rural communities have certain characteristics that have to be considered for effective implementation of health information. Moreover, physicians, nurses, physician assistants, and nurse practitioners are all in high demand, but low quantity as well. This shortage of health care personnel coupled with a lack of equipment and facilities often forces rural residents to drive long distances to receive health care and transportation to facilitate healthcare needs is often an issue.

Rural lifestyles tend to affect the health of individuals. Occupations in rural areas tend to be more dangerous as well as provide lower income than in urban areas. Studies have shown that rural individuals have higher rates of tobacco and substance abuse as well as increased levels of malnutrition or obesity. All of these lifestyle factors can result in greater instances of injury related to occupation and disease. Furthermore, rural communities tend to be small and close-knit, which is perceived as contributing to privacy issues. Some residents will not seek health care due to the fear that others in the community will know about their health issues. These concerns coupled with their busy lifestyles, i.e., focused on hard work and providing for their families, results in health care ranking low on the priority list.

\section{Telemedicine}

Telemedicine has been defined as “ . . the use of electronic information and communication technologies to provide and support healthcare when distance separates the participants.." [4]. Telemedicine programs typically consists of a central facility e.g. an Urban hospital or an academic institution and one or more remote sites such as a community hospital or a clinic [5]. Over the past few years, a small segment of research has focused on the benefits and risks of adopting and implementing information systems and technologies in the rural areas. These projects have utilized prior research methods of urban information systems and telemedicine implementations in order to tailor their studies to the unique challenges and opportunities that rural areas face. Researchers believe that information systems have the ability to enhance health care delivery but may be cost prohibitive [6].

Lindberg [7] created a study in rural Kansas to investigate the role of telemedicine in the treatment of elderly and disabled patients. This Interactive Home Health Care Program utilized the Hays Medical Center, which serves twenty-seven rural counties. Local cable systems interconnect a base station staffed by a telemedicine nurse with a modified television installed in the homes of the elderly patients. Individual patient profiles were showing improvement after six months.

The inherent challenges were technical and social aspects. Technical aspects included translating nursing protocols from hospitals to telemedicine, improving audio quality on the units, as well as software design problems that have impacted nurses [7]. Socially, nurses felt isolated while using the telemedicine methodology; however, it allowed them to tailor the treatment for each patient. To date, the program is continuing to be evaluated, but shows promising results and the ability to be applied on a broader scale.

Reddy, Purao and Kelly [8] completed a case study in the area of information technology infrastructure development for rural hospitals. Information technology in hospitals has the potential to reduce medical errors, lower costs, and improve patient care; however, many hospitals cannot afford the high costs of creating the necessary infrastructure. While rural hospitals especially have difficulty obtaining necessary resources, these areas are showing an increasing demand for health services.

This case studied a network created by three rural hospitals, which outsourced their IT needs to another larger regional hospital. The researchers interviewed eleven members of senior management at the rural hospitals as well as the IT partner hospital. Participants generally expressed a positive view of the network. The regional 
hospital and the rural hospitals received financial savings based upon cost reductions and economies of scale. Furthermore, the rural hospital benefited from the shared IT expertise, which allows them access to the regional hospital's IT staff experts.

Although both participants received cost benefits, there were indeed challenges, such as the perceived disparity between the views of customer service and the growing dependence of rural hospitals with respect to regional providers. These challenges were resolved by defining the roles and expectations of customer support participants as well as regional provider responsibilities. The rural hospitals' decisions were often heavily influenced by the provider [8].

A more recent project is "an agreement between Jackson State University in Mississippi, USA, Shiraz University of Medical Sciences in Iran, the consulting firm Oxford International Development Group, and the Jackson Medical Mall, which provides health care to the poor in Mississippi's capital." This collaboration effort will bring the Iran model of Health Houses to the citizens of Greenwood, Mississippi, one of the poorest regions of the United States, with inaccessible healthcare and high infant mortality rates. The health houses are staffed by local citizens and provide preventive care, resulting in better healthcare and fewer trips to the emergency room [9].

The common key purpose of these studies is collaboration. However, these collaborative efforts must be managed effectively. The interdependence of relationships is complex and must be detailed for all participating parties, with roles, responsibilities, and expectations clearly communicated throughout the process. Finally, a mutual understanding of the goals will establish a solid foundational partnership with the larger hospital. The collaborative model presented in this research considers these issues and addresses the method for a successful partnership.

\section{COLlaborative MODEL}

The success and sustainability of rural healthcare information systems resides in the collaboration and partnerships among cooperating participants. Increased demand for health services, need for IT infrastructure, fewer practicing physicians and medical support personnel illustrate the urgent need for a healthcare revolution. Within the practice of healthcare, referrals are usually based on personal relationships. The side-by-side working of primary-care physicians and specialist is a vital component of developing a trust. Given the cultural differences, the specialists need to have an understanding of rural culture and norms. Rural primary care physicians might face the challenge of lack of exposure to modern technology besides the patients. Thus for a successful deployment of a health information system, it is necessary to build collaboration amongst specialists, primary-care physicians, knowledge centers and technology partners [10]. In this section we propose a collaborative model for the development of health information system that would address these by effectively joining technology partners, teaching institutions, and healthcare providers (Figure 1).

The use of information systems has in general reduced medical errors, costs, and improved patient care. For instance, Decision Support Systems in medical decisionmaking have assisted with illness diagnostics, care provider interventions, retrospective quality assurance re-

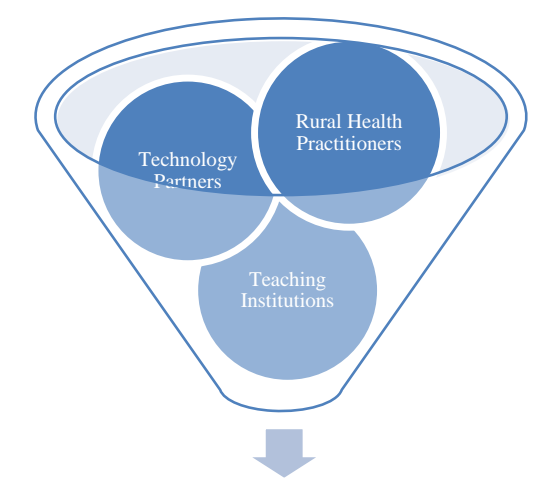

\section{RURAL HEALTH CARE TECHNOLOGY}

Figure 1. Rural Healthcare Collaborative Model

views, and previous decision critiques [11, 12]. Prior to large-scale health information systems, simple computer based electronic records were utilized to manage patient records. Major medical facilities have since migrated to large and effective health information systems. These systems have proven to enhance healthcare delivery and preventive care; thus, health information systems offer potential benefits, if implemented correctly in rural and underdeveloped communities. However, since healthcare information systems applications are designed and implemented in metropolitan areas, the necessity to meet the needs of underdeveloped regions is paramount. In this paper we present a collaborative health information systems model that can be used in rural and under-developed regions and developing regions worldwide (Figure 1).

\section{A. Rural Practitioners}

The healthcare information system will begin with selected rural practitioners and institutions and then gradually evolve to include more rural outlets. These participants will be responsible for providing electronic health records as well as clinical trial data. In addition, they will have access to the electronic health records repository for pertinent and relevant information for their rural medical practice. If they have a medical question, they will be able to access past telemedicine conferences to see if their question has been previously discussed (Figure 2).

Culture is known to influence a number of aspects of individual's healthcare, including individual health beliefs, attitude towards medical procedures and disclosure of medical information [13]. Rural practitioners would have a better familiarity with the local cultures and attitudes. Therefore the role of the rural practitioners is critical in the success of the proposed collaborative model.

The network can be used to deliver programs allowing for dissemination of new developments and research in health sciences for the rural primary care physicians and hospital staff [5]. Collaboration teaching institutions through the network would allow the rural practitioners to deliver better service to rural communities while moderating the patient attitudes towards the role of health information systems.

\section{B. Technology Partners}

The role of suitable technology is of critical importance in the success of model. With the advances in technology and increase in the patient health record data being main- 
tained, the technology partners would provide the architecture and applications necessary to facilitate the communication flow and information exchange of the network. Prior research has indicated that the awareness of technology and transmission of knowledge to health professional through telecommunication networks is effective [14].

Rapid developments in electronic healthcare systems, makes the use of such systems by healthcare providers a challenge. While the healthcare providers are experts in the area of diagnostics and treatment, they usually find the use of new technology challenging. The creation of a useful digital knowledge base is dependent on how effectively the technology is used in practice. The technology partners therefore would also be responsible for imparting training in the use of the technology. Furthermore, the technology partners will be responsible for any maintenance and updates to the information system (Figure 2). Thus the role of the technology partners would be the seamless integration of technology and the knowledge workers.

\section{Teaching Institutions}

The teaching institutions involved in the rural healthcare information system will be participants of the Rural Health Institution. These institutions will provide statistical data and analysis of their research, best practices for use of the information system, and any additional information relevant to their scholarly activities. The participating institutions will be able to access the repository to search for research participants fitting the study criteria (Figure 2).

The rural practitioners will provide electronic health care records as well as clinical trial data, while the technology partners provide the architecture and applications to facilitate the information network. The teaching institutions will maintain the data repository that stores electronic health records, clinical trial data, and the telemedicine archives. Figure 3 illustrates the flow of information and the interaction of stakeholders in the proposed model.

The information system will reside in the center of the repository. The repository will store electronic health records, clinical trial data, a telemedicine archive, and other information useful to the information system's participants. In order to access the repository, participants will be required to have a User ID and Password. This security measure will help to increase data integrity by providing information about participants that access, update, or delete information. Moreover, it will ensure privacy of information by allowing access only to those who have a registered User ID and Password.

The technology partners will be able to communicate directly to the rural practitioners and teaching institutions during support activities related to the information systems. Furthermore, the information system will include a telemedicine component, which will allow the teaching institutions to assist the rural practitioners with medical questions and situations.

\section{IMPLEMENATION CHALLENGES}

Privacy of data and interoperability are some of the challenges that rural practitioners would encounter while using general health information systems [15]. The challenge of privacy is not only for the patients, whose infor-

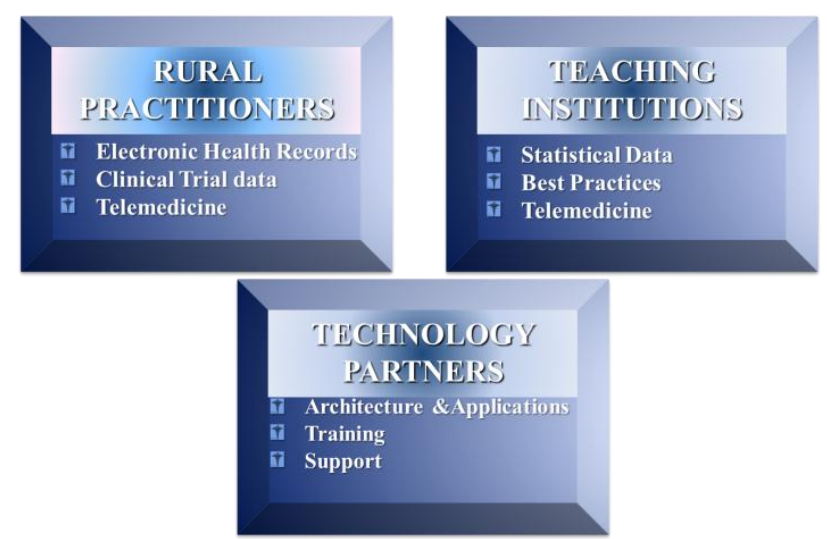

Figure 2. Roles and Responsibilities of Participants

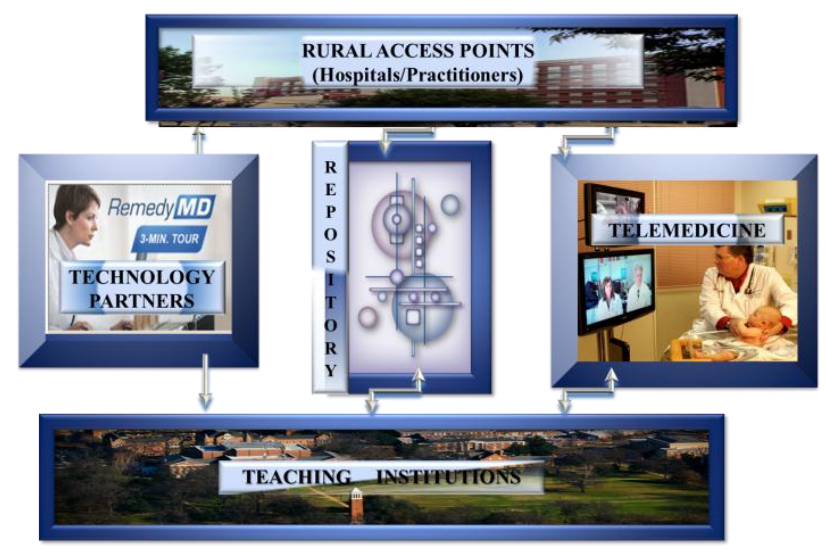

Figure 3. Communication Flow

mation is being collected, but also for the professionals who are using the system. Some patients may be hesitant to share personal information or give consent for their information to be stored in the repository. The inherent risk for physicians in using an information system with User ID and password access is the ability to trace all activities while interacting with the information system. This could discourage physicians who are concerned about insurance malpractice claims.

Developing trust in telemedicine is a challenge that has been identified in past studies. In rural settings health literacy, attitudes towards technology and gender roles could impact the adoption of health information technology [16]. When developing health information systems for use in rural areas, issues of effective user interfaces that promote willingness on the part of e-health consumers would play a critical role in developing trustworthiness of these systems [17].

Coupled with security concerns, interoperability is vital to the success of the project; thus, creating an information system that is compatible with existing systems is a necessity for long term sustainability. If the system is not capable of working with current systems or if employees have not engaged in training activities to appreciate the benefits of the system, adoption rates may be low, and the system might ultimately fail. The technology partners can help to overcome these challenge by carefully creating an information system to maximize interoperability, and provide training opportunities beyond implementation.

In our model, the technology partners, with existing expertise, in these areas would transfer that burden off the practitioners. Maintenance and upgrade of the software, 
resulting in significant cost and effort, would also reside with the technology partners. Teaching institutions would serve the role of liaisons by providing the statistical analysis data and expertise to take full advantage of the electronic medical records. This collaborative effort will create best practices for effective use of technology in rural health care.

\section{CONCLUSION}

Rural healthcare needs urgent reform and immediate action is warranted on a global scale. Technologies used in medical care have assisted with diagnostics, interventions as well as management of patient care and records. The future of such technologies as health information systems will allow health care providers and patients to manage their care more effectively with a seamless network. Hence, these systems will connect a wide area network of real-time, life-critical data. Currently, this lack of connectivity presents the issue of not providing the "best quality" care available for the patient.

Therefore, the collaborative model presented in this research can be applied to rural communities and underdeveloped regions worldwide. The suggested model effectively joins technology partners, teaching institutions, and healthcare providers. The future of this research effort will include the development of the study and testing the model for viability. The effective implementation of this health information system will change the proverbially face of rural healthcare. The success and sustainability of rural healthcare information systems resides in the collaboration and partnerships among cooperating participants. We have the technology, we have the information, and we have willing partners. It is time to reform rural healthcare and take action to provide necessary healthcare worldwide.

\section{REFERENCES}

[1] U.S. Census Bureau: Report on Urban and Rural Population by State. 2000

[2] Rosenblatt, R. and Hart, L., "Physicians and Rural America." Western Journal of Medicine, November, vol. 173, pp. 348-351, 2000.

[3] Bureau of Health Professions. "Rural Health Professions Facts: Supply and Distribution of Health Professions in Rural America." Rockville, MD: Health Resources and Services Administration. 1992.

[4] Gonzalez, E "Connecting the nation: classrooms, libraries, and health care organizations in the information age" National Telecommunications and Information Administration office of telecommunications and Information Applications. United State Department of Commerce 1995.

[5] Zollo, S, Kienzle, A. Henshaw, Z., and Crist, L., "Tele-Education in a Telemedicine Environment: Implications for Rural Health Care and Academic Medical Centers" Journal of Medical Systems vol. 23 issue 2 pp. 107-122, 1999. doi:10.1023/A:1020589219289

[6] Staggers, N., Thompson C.B., and Snyder-Halpern, R. "History and trends in clinical information systems in the United States."
Journal of Nursing Scholarship, vol. 33 pp. 75-81, 2001. doi:10.1111/j.1547-5069.2001.00075.x

[7] Lindberg, C. "Implementation of In-home Telemedicine in Rural Kansas Answering an Elderly Patient's Needs." Journal of the American Medical Informatics Association (JAMIA); vol. 4, pp. 14-17, 1997. doi:10.1136/jamia.1997.0040014

[8] Reddy, M., Purao, S., and Kelly, M. "Developing IT Infrastructure for Rural Hospitals: A Case Study of Benefits and Challenges of Hospital-to-Hospital Partnerships." Journal of the American Medical Informatics Association (JAMIA) vol.15, pp. 554-558, 2008. doi:10.1197/jamia.M2676

[9] Byrd, S. "Mississippi looks to Iran for Rural Health Care Model." Associated Press, 2010.

[10] Hilty DM, Yellowlees PM, Cobb HC, Bourgeois JA, Neufeld JD, Nesbitt TS. "Models of telepsychiatric consultation--liaison service to rural primary care." Psychosomatics. vol. 47 issue $2 \mathrm{pp}$. 152-7, 2006. doi:10.1176/appi.psy.47.2.152

[11] Brossette, S., Sprague, A., Hardin, J., Waites, K., Jones W., and Moser, S. "Association Rules and Data Mining in Hospital Infection Control and Public Health Surveillance." Journal of the American Medical Informatics Association. vol. 5, pp. 373-381, 1998. doi:10.1136/jamia.1998.0050373

[12] Hardin, J. and Raja, U., "Expert Systems and Decision Support" in Health Information Management: Concepts, Principles, and Practice, $3^{\text {nd }}$ Edition, 2009.

[13] Yellowlees P, Marks S, Hilty D, Shore JH. "Using e-health to enable culturally appropriate mental healthcare in rural areas." Telemed J E Health. vol. 14 issue 5 pp. 486-92, 2008. doi: $10.1089 / \mathrm{tmj} .2007 .0070$

[14] Puskin, D. "Telecommunications in Rural America: Opportunities and Challenges for the Health Care System." Annals of the New york Academy of Science vol. 670, pp 67-75, 1992. doi:10.1111/j.1749-6632.1992.tb26075.x

[15] Brailer, D. "Interoperability: The Key To The Future Health Care System. " Health Affairs, 10.1377/hlthaff.w5.19., 2005. doi:10.1377/hlthaff.w5.19

[16] Wathen, N. "I Try to Take Care of it Myself. How rural women search for health information" Qualitative Health Research. vol 17 issue 5 pp. 639-651, 2007. doi:10.1177/1049732307301236

[17] Furling, A. and Lee, S., "The influence of user-interface usability on rural consumers' trust of e-health services." International Journal of Electronic Healthcare, vol. 2 issue 4, 2006

\section{AUTHORS}

U. Raja (uraja@cba.ua.edu) is with The University of Alabama, Tuscaloosa, AL 35487 USA.

D.J. McManus (dmcmanus@cba.ua.edu) is with The University of Alabama, Tuscaloosa, AL 35487 USA.

J. M. Hardin (mhardin@ cba.ua.edu) is with The University of Alabama, Tuscaloosa, AL 35487 US.

B. C. Haynes (bchaynes@uab.edu) is with The University of Alabama School of Medicine, Birmingham, AL 35294 USA.

This article is an extended version of a paper presented at the Interdisciplinary Conference of AHLiST 2010 Conference, June 2010, Madrid, Spain. Received May $10^{\text {th }}, 2011$. Published as resubmitted by the authors June $9^{\text {th }}, 2011$. 